\title{
La ilustración italiana: defensa de los derechos humanos. Cesare Beccaria (1738-1794) y Pietro Verri (1728-1797)
}

\author{
Mariapia LAMBERTI \\ Universidad Nacional Autónoma de México
}

El siglo XVIII fue para Italia un siglo de lento despertar. La época del Renacimiento y del Barroco, que significaron la formación y el fortalecimiento de las grandes monarquía europeas, destinadas a expandir su poderío allénde la mar Océana, finalmente surcada a lo ancho y lo largo por lo redondo del planeta, vieron la decadencia de Italia por lo que atañe a su constitución política. Bien había temido y profetizado Machiavelli que el abuso de las armas mercenarias, las luchas de las facciones internas a las ciudades, y la lucha de las ciudades internas a la Península acabarían con la libertad de Italia. Y así fue. A la invasión francesa sucedió la dominación española, y a ésta la dominación austriaca. Las grandes potencias se repartieron a menudo el territorio italiano como parte del botín de sus guerras privadas. Pero Italia no se vio unificada ni siquiera por obra de las conquistas: permaneció fraccionada y dividida en múltiples estados, en territorios virreinales o en monarquías y principados independientes en la medida en que pueda considerarse independiente un estado minúsculo y sin fuerzas ligado por intereses múltiples a un poderoso. La independencia y unidad no podía venir sino desde las fuerzas internas, por obra de una profunda convicción.

En la primera mitad del siglo XVIII esta conciencia de unidad e independencia está todavía lejos de manifestarse. Causa o efecto de ello - las opiniones son divergentes-, una cierta modorra intelectual, que con honrosas excepciones abarca la plenitud de la época barroca y se prolonga por buena parte del Siglo de las Luces. Las luces del siglo vendrán de fuera, de Francia y de Inglaterra, es bien sabido; pero ¿hubo luces propiamente italianas, contribuyó el intelecto italiano a ilustrar el siglo de la Ilustración?

La primera mitad del siglo presenta un panorama significativo, si lo contemplamos desde la perspectiva, que ya fue del gran De Sanctis, de la formación progresiva del espíritu nacional. Los italianos no son tales aún, 
pero manifiestan, en el siglo del cosmopolitismo, la inquietud de reconocerse pueblo, etnia: investigando el pasado histórico en términos ya unitarios. La historia de la literatura es el gran tema; después del primer intento de recopilación cronológica de la poesía italiana (Istoria della volgar poesia, 1697 y Bellezze della volgar poesia, 1712) de Giovan Maria Crescimbeni, Gian Vincenzo Gravina publica un Della ragion poetica en 1712 , en la que teoriza en términos clasicistas sobre la literatura, y deja al mismo tiempo una panorámica de las letras nacionales. Italia se reconoce en sus poetas; basta examinar los títulos de las obras de literatos oscuros o más famosos: Idea della storia dell'Italia letterata (1723) escribe un Giacinto Gamma, y se considera la primera historia literaria de Italia; más adelante en el siglo, Giovan Maria Mazzuchelli empieza a recopilar un diccionario biográfico (1753-1763) que titula Gli scrittori d'Italia; y finalmente, Girolamo Tiraboschi (1731-1794) nos lega trece volúmenes de una Storia della letteratura italiana todavía considerada capital a finales del siglo XIX e inicios del XX. El nombre de Italia y el adjetivo italiano se aplicaban libremente al ámbito literario.

Tiraboschi empezaba su erudito recorrido desde los etruscos. El apasionamiento por las antigüedades - típico por demás en el siglo de las primeras investigaciones arqueológicas - cobra entre los eruditos de la península un valor análogo al de las investigaciones literarias: es una búsqueda de identidad, que se ahonda en las memorias históricas. Ludovico Antonio Muratori (1672-1750) es el máximo exponente de esta carrera tan dieciochesca hacia la investigación anticuaria. Polígrafo ilustre, teórico del "gusto" (Della perfetta poesia italiana, 1706; Riflessioni sopra il buon gusto nelle scienze e nelle arti, 1708), es conocido sobre todo por los 28 volúmenes in folio de los Rerum italicarum scriptores, recopilación de documentos datados entre el siglo VI y el XVI, las Antiquitates italicae Medii Aevi (1739-1743), y finalmente los Annali d'Italia (1744-1749), nuestra primera historia nacional, construida sobre bases filológicas.

La recopilación histórica es madre de la crítica política; y si el propio Muratori llega a la teorización de un "despotismo ilustrado" ante litteram, afirmando que el principado no ha sido inventado "para conseguir el bien del solo príncipe, sino principalmente para conseguir el bien de la República, o sea para procurar la felicidad de los pueblos súbditos del principado", ${ }^{1}$ Pietro Giannone (1676-1749) llega mucho más allá, luchando con

\footnotetext{
${ }^{1}$ Encuentro esta cita, que traduzco, en Floccia:1967, 287. La cita viene del tratado Della pubblica felicità de Muratori.
} 
sus escritos y su vida (padeció excomunión y cárcel, muriendo por cierto en prisión) contra el poder papal, analizando las luchas entre Estado e Iglesia en su Istoria civile del regno di Napoli (1723), y luego en los Discorsi politici sopra gli annali di Tito Livio, donde se anticipa a Rousseau criticando los dogmas cristianos manipulados para reducir a los hombres a la sumisión, y reivindica el derecho al libre pensamiento, pregonando un regreso de la comunidad cristiana al espíritu evangélico.

En armonía con esta pasión por la documentación histórica y la visión global y crítica del pasado, la luz brillante de este primer medio siglo no podía sino gravitar en el ámbito de la Historia: y será la del atribulado y oscuro filósofo Gian Battista Vico, solitario e incomprendido, pero que dejaría tras de sí la estela refulgente del historicismo, la máxima aportación del genio itálico a la filosofía occidental. Vico trasciende el siglo de las luces, y reverbera las suyas sobre el Romanticismo y la época moderna. Su Scienza nuova $(1725 ; 1744)$ rescata el quehacer humano $-\mathrm{y}$ el ser-, revaluando la infancia, la fantasía, y la creación poética como momento del espíritu y fundamental tapa evolutiva de las Naciones.

La segunda mitad del siglo está dominada, en el ámbito internacional, por la aparición de la Encyclopédie (1751-1780), alrededor de la cual gravita la obra de todos los philosophes que han dejado su nombre como sello de la época. Analizar el presente como fruto torcido del pasado para preparar - reclamar- el cambio para el inmediato futuro: éste, en síntesis, el fin primario del movimiento enciclopédico. La Ilustración ha sido un movimiento impaciente, que no quería ni podía esperar los largos tiempos de los inevitables recursos viquianos.

La difusión de las nuevas actitudes críticas en Italia se debió principalmente a la obra de traducción. El mismo Condillac, que residiera en el ducado de Parma entre 1758 y 1767, se ocupó de la traducción de la Enciclopedia; un sacerdote, Francesco Lugano (1743-1806), fue el primer difusor de las ideas sensistas de Locke y Condillac, aunque sincretizadas con los presupuestos dogmáticos del cristianismo. La difusión del sensismo marcó profundamente la llustración italiana, que en la segunda mitad del siglo XVIII se caracterizó por las polémicas alrededor de la naturaleza del arte y de lo bello, y por las acendradas discusiones sobre la naturaleza de la lengua italiana, que después de la pasión por la reconstrucción histórica y literaria de la primera mitad del siglo, venía a completar la búsqueda de identidad que hemos tratado de reconocer en los movimientos intelectuales dieciochescos en la península. Destacan en estas discusiones y polémicas los polígrafos Melchiorre Cesarotti (1730-1808) que dejó varias obras sobre el deleite del arte y la literatura y la naturaleza de 
las lenguas, y tradujo los Cantos de Ossian; Francesco Algarotti (17121764), viajero según la moda del siglo y amigo de personajes ilustres (entre ellos Voltaire), que, además de tratados sobre el arte militar y sobre la lengua militar italiana, divulgó un Newtonianismo per le dame; Saverio Bettinelli (1718-1808), jesuita, el alma más intolerante de las restricciones mentales y rebelde en la difusión de las nuevas ideas, historiógrafo desacralizador en sus críticas literarias. Por cierto, es curioso notar cómo en el siglo XVIII prevalece entre los que se ocupan de literatura una crítica despiadada contra el "mal gusto" y "rudeza" de Dante Alighieri. Bettinelli no es la excepción, y fue Gaspare Gozzi, el veneciano hermano del comediógrafo Carlo, quien se encargó de la defensa del máximo poeta de Italia. Giuseppe Baretti (Turín, 1719-Londres, 1789), en fin, con su publicación periódica La Frusta Letteraria, con el seudónimo de Aristarco Scannabue (algo así como... "degüellabueyes") toma como pretexto la crítica literaria para lanzarse también contra la necedad del siglo. Pero su preocupación prioritaria sigue siendo el estado de las letras italianas, la naturaleza demasiado arcaizante y farragosa de la lengua italiana, la funcionalidad de estos dos instrumentos príncipes de la evolución intelectual para la sociedad italiana...

Un panorama interesante, pero excesivamente provinciano y solipsista, aunque los particularismos se justifiquen por las circunstancias, y sean portadores de futuros desarrollos. No es propósito de esta breve panorámica tocar las producciones literarias, ricas también de implicaciones intelectuales: dejamos de lado intencionalmente la mención de los grandes autores: Goldoni, el renovador del teatro, el moralista Parini y el genio de final de siglo y fustigador de la tiranía, el tragediógrafo Vittorio Alfieri. Pero, ¿dónde reconocer la aportación universal, supra-provinciana, de la Ilustración italiana al mundo?

Ya desde la época de Vico, a las investigaciones históricas y las teorizaciones estéticas, literarias y lingüísticas, se había acompañado otra serie de reflexiones e investigaciones, acorde con la más antigua y tradicional cultura itálica: las reflexiones jurídicas. Vico mismo había competido (sin éxito) por una cátedra de jurisprudencia en Nápoles, y había publicado en 1720 una Sinopsi del diritto universale, dos libros De uno universi iuris principio et fine uno, un tratado sobre la naturaleza universal del derecho, como reza el título, y otro De constantia iurisprudentis. Otros pensadores e intelectuales promovieron reformas de carácter económico y jurídico en los Estados de pertenencia: el salernitano Antonio Genovesi (1713-1769), catedrático en Nápoles, e inspirador de las reformas actuadas bajo el reinado de Carlos III en aquel reino; Ferdinando 
Galiani (1728-1787), del mismo reino borbónico, escribió un tratado sobre la moneda, y otro sobre el comercio; pero sobre todo hay que recordar, en la línea jurídica, a los napolitanos Mario Pagano (1748-1799), que muriera durante la feroz represión del ' 99 después del brote revolucionario del reino de Nápoles, y el joven jurista Gaetano Filangieri (17521788), quienes propugnaron por una reforma profunda de la legislación penal, que comprendía la abolición de la tortura y de la pena de muerte; sobre todo el último intentó tratar la jurisprudencia como una ciencia, y entre las reformas que proponía, además de la abolición de los privilegios feudales y la reforma penal, estaba el modernísimo concepto de la enseñanza gratuita por parte del Estado.

Pero estas ideas gestadas en el sur profundo y perennemente reaccionario, encontrarían desarrollo, luz y difusión internacional en el norte. El norte de Italia pertenece al Imperio Austriaco, siempre propicio al orden social y a la moderación, y abierto a las novedades. Un grupo de jóvenes entusiastas de las nuevas ideas francesas, siguiendo la moda de los tiempos, funda en Milán una publicación periódica cuyo nombre refleja otra moda de los tiempos, destinada también a perdurar: Il Caffè. Se llaman Pietro (1728-1797) y Alessandro (1741-1816) Verri; y Cesare Beccaria (1738-1794). El periódico tiene breve vida (1764-1766) porque la censura austriaca no lo pierde de vista, y encuentra pretextos para detener su publicación. Pero las ideas allí debatidas se difundirán también en escritos más orgánicos.

Pietro Verri fue el iniciador y animador de la revista y de una Academia (no hay que olvidar que el siglo XVII había sido en Italia el siglo de las Academias, y la costumbre no se había extinguido) que llamó "dei Pugni" o sea de los puñetazos. Seguidor del utilitarismo y sensismo de Condillac y Helvétius, escribió, adelantándose a Freud, que la dinámica propulsora del ser humano es el principio del placer y rechazo del dolor: Discorso sull'indole del piacere e del dolore (1773). Su campo fue la economía: proyectó una reforma fiscal para el gobierno austriaco, y escribió Meditazioni sull'economia politica (1771). Fue, en política, absolutista y luego constitucionalista. Su hermano Alessandro fue más inclinado hacia la literatura: traductor de Hamlet y Otello de Shakespeare, abandonó el círculo milanés (manteniendo un interesante epistolario con su hermano Pietro) para ir a vivir en Roma; ha entrado en la historia literaria de Italia con las prerrománticas Notti romane al sepolcro degli Scipioni. En su época milanesa, colaborando en la revista, con brillantes artículos demostró la necesidad de renovar los sistemas jurídicos vigentes en Europa. 
Pero fue Beccaria quien supo dar un viraje del todo especial a la corriente jurisprudencial y a las meditaciones sobre las reformas urgentes del sistema penal. Entre 1664 y 1766 (son los dos años de vida de la revista $I l$ Caffè) redactó un breve y compendioso tratado, que llamó a la latina Dei delitti e delle pene. ${ }^{2}$ Con sencilla lucidez, Beccaria examina los procedimientos codificados en uso en su época (pero, jay!, todavía comunes hoy día) para castigar los crímenes. Su defensa de los que hoy llamaríamos derechos humanos es plana y serena, convincente: no hace recurso a la vehemencia de la indignación, sino a la persuasión: torturar es inútil, matar por ley no tiene posible justificante jurídica. El castigo debe propiciar de un lado la recuperación del criminal, del otro el escarmiento público: ¿y qué mayor escarmiento, pregunta Beccaria, que el contemplar a los que dañaron la sociedad trabajando para beneficio de la misma? La propuesta de Beccaria, que examina en 46 apartados y una conclusión, de un punto de vista práctico y teórico, todas las facetas de las leyes y de los procedimientos, ${ }^{3}$ se concentra en dos puntos: la gravedad del crimen se debe juzgar según la entidad del daño provocado a la sociedad ("l'unica vera misura dei delitti è il danno fatto alla nazione" [Beccaria: 1995, 22]); el trabajo obligado en beneficio de la sociedad ofendida es el castigo más ejemplar, más lógico y más beneficioso.

${ }^{2}$ En la edición que se ha utilizado (1995) para el presente trabajo del tratado de Cesare Beccaria, Dei delitti e delle pene, volumen que además de las notas biográficas y del ensayo introductivo incluye los textos colaterales de las polémicas suscitadas por la obra en toda Europa, sobre las 672 páginas totales (más las XXXIX de la introducción) el texto ocupa sólo de la página 1 a la 104.

${ }^{3}$ Traslado y traduzco aquí el título de los apartados: I. Origen de las penas; II. Derecho de castigar; III. Consecuencias; IV. Interpretación de las leyes; V. Oscuridad de las leyes; VI. Proporción entre crímenes y penas; VII: Errores en la medida de las penas; VIII. Subdivisión de los crímenes; IX. De la honra; X. De los duelos; XI. De la tranquilidad pública; XII. Finalidad de las penas; XIII. De los testigos; XIV. Indicios, y formas de juicio; XV. Acusaciones secretas; XVI. De la tortura; XVII Del fisco; XVIII. De los juramentos; XIX. Prontitud de la pena; XX. Violencias; XXI. Penas de los nobles; XXII Hurtos; XXIII. Difamación; XXIV. Ociosidad; XXV. Destierro y decomisos; XXVI. Del espíritu de familia; XXVII. Suavidad de las penas; XXVIII. De la pena de muerte; XXIX. De la captura; XXX. Juicios y prescripción; XXXI. Crímenes de comprobación dificil; XXXII. Suicidio; XXXIII. Contrabando; XXXIV. De los deudores; XXXV. Asilo; XXXVI. De la recompensa [por la captura del criminal]; XXXVII. Atentados, cómplices, impunidad; XXXVIII. Interrogaciones sugerentes, declaraciones; XXXIX. De un género particular de crímenes [religiosos]; XL. Falsas ideas de utilidad; XLI. Cómo prevenir los crímenes; XLII. De las ciencias; XLIII. Magistrados; XLIV. Recompensas; XLV. Educación; XLVI. De las gracias; XLVII. Conclusión. 
Tortura y pena de muerte no sólo ofenden a la humanidad, sino también el sentido común, porque de nada sirven:

La morte è ella una pena veramente utile e necessaria per la sicurezza e il buon ordine della società? La tortura e i tormenti sono eglino giusti, e ottengono eglino il fine che si propongono le leggi? Qual è la miglior maniera di prevenire i delitti? (30).

Uno dei piú grandi freni dei delitti non è la crudeltà delle pene, ma l'infallibilità di esse [...]. La certezza di un castigo, benché moderato, farà sempre una maggiore impressione che non il timore di un altro piú terribile, unito con la speranza dell'impunità (59).

[...] la sperienza di tutti i secoli, ne' quali l'ultimo supplicio non ha mai distolti gli uomini determinati d'offender la società [...] (63).

La tortura y la pena de muerte no pueden tener ninguna base jurídica:

Qual può essere il diritto che si attribuiscono gli uomini di trucidare i loro simili? Non certamente quello da cui risulta la sovranità e le leggi (62).

La jurisprudencia (justicia humana) debe separar el concepto de crimen del concepto de pecado (fuera del alcance humano). Los presupuestos de la ley, como las relaciones entre los hombres deben ser igualitarios:

[...] alcunini pensarono che la gravezza del peccato entrasse nella misura dei delitti. La fallacia di questa opinione risalterà agli occhi di un indifferente esaminatore dei veri rapporti tra uomini e uomini e tra uomini e Dio. I primi sono rapporti di uguaglianza (23).

La historia demuestra que la pena de muerte es y se acompaña con la barbarie:

I paesi e i tempi dei piú atroci supplicii furon sempre quelli delle piú inumane azioni $[\ldots](60)$.

La justicia debe fundamentarse sobre la lógica de los derechos:

La sicurezza della propria vita è un diritto di natura, la sicurezza dei beni è un diritto di società (74). 
Y no falta una lúcida visión de la injusticia social que subyace a estos bárbaros excesos:

[...] la miseria, o voluta o tollerata dalle leggi, che hanno sempre favorito i pochi ed oltraggiato i molti... (61).

La repercusión fue inmediata, y como bien se puede imaginar, en un principio la reacción adversa fue la más evidente. Se definió a Beccaria como "el Rousseau de los italianos" (y seguramente la comparación no se hacía en términos honrosos), como "socialista" (y fue la primera vez que esta palabra se empleó como arma contra alguien). Si alguna concesión se le hacía, era para aceptar los contenidos de su propuesta, no las bases ideológicas: hasta su enemigo inicial, Carli, terminó definiendo el opúsculo "il primo libro che sia stato scritto in Italia in favore dell'umanità". Pero rechazaba los principios igualitarios que lo habían inspirado.

Pero otra suerte tuvo la obra de Beccaria allende los Alpes. En 1765 se había terminado la redacción de la Enciclopedia, e iniciaba el "cisma rousseauiano". Los principios inspiradores de Beccaria, que, educado por los jesuitas como todo joven de familia noble milanesa, se había posteriormente alimentado con las lecturas de las Lettres persanes de Montesquieu, del De l'ésprit de Helvétius, del Contrat social y la Nouvelle Hélö̈se del ginevrino, sonaban muy cercanos a la fuente inspiradora, y no predisponían a su aceptación. Pero la fuerza del tratado era inmensa: Melchor Grimm afirmó: "Il serait à désirer que tous les législateurs de l'Europe voulussent prendre les idées de M. Beccaria en considération et remédier à la barbarie froide et juridique de nos tribunaux". ${ }^{4}$

La difusión del tratado es inmediata. En 1766 los ilustrados franceses invitan al joven noble milanés a París para que diserte sobre sus ideas y propuestas. Beccaria tiene una frágil constitución nerviosa (que heredará su ilustre nieto Alessandro Manzoni), sufre de temores y manías. Acepta el viaje con renuencia, pero es incapaz de llevar a término los compromisos que la visita le impone. No importa, el fracaso del encuentro con la intelectualidad más prestigiosa de Europa no merma la importancia de su tratado. Los philosophes discuten los principios rectores y analizan, acaso con cierta duda, las posibles consecuencias de las reformas propues-

${ }^{4}$ La mención de Gianrinaldo Carli y de Grimm, con las dos frases citadas, la encuentro en las pxiv y xxi respectivamente, de la Introducción de Franco Venturi a Dei delitti e delle pene, imprescindible página de estudio sobre Beccaria. Ésta ha sido la fuente de otros datos y noticias que comento en mi trabajo. 
tas, pero aceptan la base humanitaria que rige el tratado. Si es cierto que la magistratura francesa asi como la inglesa estaba terriblemente reacia frente a la posibilidad de reformas, sin embargo después del ' 70 el genio de Voltaire avala las propuestas de Beccaria y aboga por el reformismo. El Abad Malby pone el dedo sobre la desigualdad social, sobre el mismo principio de la propiedad como base de la condición del crimen y del castigo. Glosando a Beccaria, llega a afirmar que la reforma penal no puede ser posible sin una profunda reforma de la sociedad.

El éxito se demuestra también a través de las condenas: Dei delitti e delle pene es incluido en el Índice en 1766, de inmediato. Pero dos años después en el mismo Estado Pontificio se discute y comenta la obra. En 1777 la Inquisición madrileña condena el libro. Pero su contenido es conocido y apreciado por Meléndez Valdés y Jovellanos.

Sin trabas el éxito en los países reformados: en 1767 la obra de Beccaria se traduce en Londres, y tendrá profunda influencia sobre William Blackstone, teórico de la ley en Inglaterra, y sobre Geremy Bentham. En 1775, en la puritana Suiza, la obra recibe el primer reconocimiento oficial, suscitando los entusiasmos protestantes.

Pero el éxito mayor de Beccaria fueron los resultados efectivos de su obra.

En Alemania, Karl Ferdinand Hommels introduce, entusiasta, la obra de Beccaria (al que llamaba su "Sócrates"), y se gana el apodo de "Beccaria Alemán"; será artífice con sus ideas de la profunda transformación del estado austriaco en la época de María Teresa y José II. En la misma Austria, Joseph von Sonnenfels inicia la batalla decisiva para la abolición de la tortura en los estados de los Habsburgo. Y en 1786, benemérito entre todos los estados europeos, el Gran Ducado de Toscana, bajo el principado de Leopoldo II sanciona la abolición de la pena de muerte. Catalina II de Rusia, la gran reformadora, invitó al ilustrado italiano a visitar su corte, reconociendo en él la persona indicada para ayudar a la transformación de su imperio en su proyecto de política interna reformista e ilustrada; inútil decir que Beccaria rehusó, presa de sus temores, pero la gran Emperatriz lo "plagió" fielmente en su Nakaz. Y del otro lado del mundo, el presidente Jefferson de los Estados Unidos meditó profundamente la obra del conde italiano.

Cuando estalla el movimiento revolucionario en París, en 1789, la obra de Beccaria se traduce con una dedicatoria a los Estados Generales en la esperanza que en la nueva Francia que se está gestando se apliquen las ideas del libro: escalofriante contraste con la embriaguez de sangre que caracterizaría el momento más dramático del movimiento que se inicia- 
ba. Más aterrador pensar que en 1791, en la Asamblea Nacional, durante la discusión sobre el nuevo código penal, el mismo Robespierre se lanzara contra la pena de muerte y la barbarie judiciaria. Pero el nombre de Robespierre, gigante de la Historia si lo hubo, queda ligado a una página terrible, mas transitoria, mientras que el de Beccaria resurge después del Terror, y no ha perdido vigencia ni actualidad en la lucha contra la barbarie sanguinaria de los hombres disfrazada de justicia.

Pero en esta batalla participó también Pietro Verri. Hombre más equilibrado, y comprometido con sus encargos administrativos del Imperio austriaco, escribió, en "refuerzo" a las ideas del amigo, en 1777 un extraño trabajo, muy novedoso para la época, que sólo se publicaría en 1804, después de su muerte, pero destinado a darle, a la sombra de Beccaria, fama imperecedera. Lo llamó Osservazioni sopra la tortura. ${ }^{5}$ Verri exhuma -allí estriba la novedad - las actas de un tremendo proceso que se llevara a cabo en Milán durante la peste de 1630. Las autoridades españolas (Milán era entonces parte del Reino de España), preocupadas por la creciente inquietud popular a raíz de la carestía y la peste, favorecieron la creencia que la mortandad fuera difundida arteramente por individuos criminales movidos por oscuras razones, los untori, "untadores" que embadurnaban puertas y paredes con ungüentos pestíferos: se crearía así unos fáciles chivos expiatorios. El furor popular identificó como untori a unos infelices particulares, que fueron apresados, torturados sin misericordia y finalmente ajusticiados de la manera más horrible. En el lugar de su suplicio se elevó, y permaneció hasta 1778 , una columna conmemorativa, conocida como Colonna infame, la columna de la infamia.

Verri, con una técnica que desarrollarán en el siglo Xx Leonardo Sciascia (La scomparsa di Maiorana, La strega e il capitano, I pugnalatori), Mario Vargas Llosa (Historia de Maita) y Gabriel García Márquez (Noticias de un secuestro), transcribe documentos reales, las actas del proceso, comentándolas, transformándolas en algo vivo, en la narración escalofriante de unos hechos. Verri se mantiene en una frialdad absoluta. Ni una vez frente a aquellos horrores se deja ir a un comentario de piedad humana, sino sólo a la indignación frente a la absurdidad y la insensatez del hecho y de los métodos. Lo que le interesa es demostrar el fracaso clamoroso de aquellas técnicas del dolor provocado. Torturar es inútil porque no lleva a los resultados deseados. La justicia (la jurispru-

${ }^{5}$ En la edición utilizada (1998) de las Osservazioni sopra la tortura de Pietro Verri, el texto ocupa de la página 49 a la última, 143. Se trata aquí también de un texto muy breve. 
dencia) es la gran perdedora: aquellos infelices torturados eran inocentes, pero dispuestos a admitir lo que fuera con tal de suspender el dolor. Y la lectura - dificil, a menudo intolerable - se transforma en algo mucho más persuasivo que si fuera acompañada con inflamadas palabras de persuasión.

Mas la trayectoria de esta noble lucha no había terminado. Giulia, la hija de Cesare Beccaria, había contraído un matrimonio arreglado por la familia, con un anciano gentilhombre de la provincia milanesa, de apellido Manzoni. El niño que nació de esta unión llevó el apellido Manzoni, pero la sangre que corría en sus venas era de la familia Verri (Pietro y Alessandro tenían un hermano menor, Giovanni, que también frecuentaba la casa de Beccaria), y el nombre, Alessandro, lo demostraba. Alessandro Manzoni (1785-1873) estaba destinado a ser el prócer literario — si se me permite el retruécano- de la futura nación italiana. Más que patriota, ideólogo de la unidad e independencia italiana, Manzoni se preocupó de cortar el nudo gordiano de la secular cuestión de la lengua no sólo pregonando su renovación, sino renovando de hecho la lengua con su obra cumbre, I promessi sposi, dejando como herencia a los italianos un instrumento real de unidad, una lengua viva, o sea capaz de evolución.

Pero Manzoni tuvo otra benemerencia con respecto a las letras italianas. Fue, después de Dante Alighieri, nuestro verdadero y profundo escritor católico. Una luz nueva vino con él a reverberar sobre la literatura italiana: la de una religión sentida, intransigente y precisa en su contexto dogmático. En la tierra que hospedaba el Papado, la religión a menudo había sido un objeto controversial, a menudo sustituida por un "paganismo" manierista, a menudo vivida sólo como dimensión individualista y heterodoxa. Manzoni fue en su juventud racionalista ateo, y en la madurez, en su búsqueda de Dios, pasó por la intransigencia calvinista y por el rigorismo jansenista, que jamás dejó de matizar su final catolicismo. Nuestro principal escritor romántico escribió unas Osservazioni sulla morale cattolica $(1819 ; 1855)$ que quedan como un hito en el pensamiento religioso italiano. $Y$ este mismo rigorismo religioso aplicó Manzoni en su obra maestra, y en un apéndice de la misma que redactó entre la primera edición (Fermo e Lucia, 1823) y la segunda edición de su novela cumbre, ya con el título definitivo de I promessi sposi, en 1840: se trata de la Storia della colonna infame, aparentemente una aclaración de un episodio central de la novela. En realidad se trata de una repetición del estudio que ya Pietro Verri había hecho de los documentos procesales del juicio (o de lo que quedaba de ellos, en su gran parte perdidos) contra los supuestos untori, en los años del siglo XVII en que se desarrolla la narración 
de las travesías de los prometidos (1628-1631). Manzoni agrega lo que faltaba al estudio del ilustre tío y al tratado del famoso abuelo: la perspectiva moral en la condena de las prácticas procesales violatorias de la integridad humana. Manzoni desmantela toda posible disculpa de los jueces que decidieron (con decisión consciente) aplicar el monstruoso método de interrogación que las mismas leyes escritas y comentadas por innumerables juristas a través de los siglos consideraban, si permitido, inseguro, contraproducente, abierto al riesgo de sadismos inútiles; que decidieron (con decisión consciente) creer en lo que no era creíble, en un delito "física y moralmente imposible"; que cedieron no a la ignorancia de sus tiempos y al permiso legal de aplicar la tortura, sino a las pasiones perversas del ánimo: rabia, miedo, conveniencia. Manzoni pone el hombre frente a su conciencia y frente a Dios: la voz de la razón que permite reconocer lo absurdo de una circunstancia, no es la sola voz que en su corazón el hombre puede escuchar; hay también, más poderosa, la voz que le hace reconocer la iniquidad, que le indica "las verdaderas y eficientes causas" de sus actos inicuos. La finalidad educativa y moral del renovado examen de aquel horror, el deseo de ir más allá de lo que había hecho su ilustre predecesor, es proclamado en voz alta:

Ma dalla storia, per quanto possa esser succinta, d'un avvenimento complicato, di un gran male fatto senza ragione da uomini a uomini, devono necessariamente potersi ricavare osservazioni piú generali, e d'una utilità, se non cosí immediata, non meno reale. ${ }^{6}$

Se concluye así esta prodigiosa triada que universaliza la Ilustración italiana: una ilustración altamente humanitaria, hondamente moral, en tres brevísimos textos: una luz que no se apaga, contribución poderosa al lento progreso de la civilización humana.

${ }^{6}$ La Storia della colonna infame ocupa de la página 961 a la 1052 en Alessandro Manzoni, Opere (1953). Se trata otra vez de un escrito de dimensiones reducidas. Tomo las dos breves citas de la 962, perteneciente a la Introducción, donde el autor expone con claridad y su inigualable precisión de palabra su intención de elevado moralismo. El estilo de Manzoni es a tal grado rotundo, tan perfecta la expresión y concatenación de conceptos, que hace casi imposible recortar breves frases: la tentación es transcribir fielmente todo el texto. 
Obras citadas

BecCaria, Cesare. 1995. Dei delitti e delle pene, a cura di Franco VENTURI. Turín: Einaudi.

FlocCIA, Giuseppe. 1967. Storia della letteratura italiana. Nápoles: Loffredo.

MANZONI, Alessandro. 1953. Opere, a cura di Riccardo BACCHELLI. Milán / Nápoles: Ricciardi.

VERRI, Pietro. 1998. Osservazioni sopra la tortura. Introd. e note di Giulio CARNAZZI. Milán: Rizzoli. 